\title{
On A-energy and S-energy of certain class of graphs
}

\author{
Harishchandra S. RAMANE \\ Department of Mathematics \\ Karnatak University \\ Dharwad, India \\ email: hsramane@yahoo.com \\ B. PARVATHALU \\ Department of Mathematics \\ Karnatak University's \\ Karnatak Arts College \\ Dharwad, India \\ email: bparvathalu@gmail.com
}

\author{
K. ASHOKA \\ Department of Mathematics \\ Karnatak University \\ Dharwad, India \\ email: ashokagonal@gmail.com \\ Daneshwari PATIL \\ Department of Mathematics \\ Karnatak University \\ Dharwad, India \\ email: daneshwarip@gmail.com
}

\begin{abstract}
Let $A$ and $S$ be the adjacency and the Seidel matrix of a graph $G$ respectively. A-energy is the ordinary energy $E(G)$ of a graph $G$ defined as the sum of the absolute values of eigenvalues of $A$. Analogously, $S$-energy is the Seidel energy $E_{S}(G)$ of a graph $G$ defined to be the sum of the absolute values of eigenvalues of the Seidel matrix $S$. In this article, certain class of $A$-equienergetic and $S$-equienergetic graphs are presented. Also some linear relations on $A$-energies and $S$-energies are given.
\end{abstract}

Computing Classification System 1998: G.2.2

Mathematics Subject Classification 2010: 05C50

Key words and phrases: graph energy, Seidel energy, equienergetic graphs, Seidel equienergetic graphs, energy linear relations, graph products 


\section{Introduction}

Let $G$ be a simple, finite and undirected graph of order $n$ with vertex set $\mathrm{V}(\mathrm{G})=\left\{v_{1}, v_{2}, \ldots, v_{n}\right\}$. The adjacency matrix $A=\left[\mathrm{a}_{\mathrm{ij}}\right]$ of $\mathrm{G}$ is a square matrix of order $n$ whose $(i, j)$-th entry $a_{i j}=1$ if $v_{i}$ and $v_{j}$ are adjacent and 0 otherwise. The eigenvalues $\theta_{1}, \theta_{2}, \ldots, \theta_{n}$ of $A$ are called the $A$-eigenvalues of $G$ and their collection is called the spectrum or $A$-spectrum of G. If $\theta_{1}, \theta_{2}, \ldots, \theta_{k}$ are the distinct $A$-eigenvalues of $G$ of order $n$ with respective multiplicities $m_{1}, m_{2}, \ldots, m_{k}$, then the $A$-spectrum of $G$ is denoted by

$$
\operatorname{Spec}(G)=\left(\begin{array}{cccc}
\theta_{1} & \theta_{2} & \cdots & \theta_{k} \\
m_{1} & m_{2} & \cdots & m_{k}
\end{array}\right) \text {, where } \sum_{j=1}^{k} m_{j}=n .
$$

In 1966 J. H. van Lint and J. J. Seidel introduced real symmetric $\{0, \pm 1\}$ matrix called the Seidel matrix $S$ is defined as $S=J-I-2 A$, where $J$ is the matrix of order $n$ whose all entries are equal to 1 and I is the identity matrix of order $n$. The eigenvalues $\lambda_{1}, \lambda_{2}, \ldots, \lambda_{n}$ of $S$ are called the Seidel eigenvalues or $S$-eigenvalues of $G$ and their collection is called the Seidel spectrum or $S$ spectrum of $G$. If $\lambda_{1}, \lambda_{2}, \ldots, \lambda_{k}$ are the distinct $S$-eigenvalues of $G$ of order $n$ with respective multiplicities $m_{1}, m_{2}, \ldots, m_{k}$, then the Seidel spectrum or $\mathrm{S}$-spectrum of $\mathrm{G}$ is denoted by

$$
\operatorname{Spec}_{S}(G)=\left(\begin{array}{cccc}
\lambda_{1} & \lambda_{2} & \cdots & \lambda_{k} \\
m_{1} & m_{2} & \cdots & m_{k}
\end{array}\right) \text {, where } \sum_{j=1}^{k} m_{j}=n .
$$

The number of positive and negative $A$-eigenvalues of $G$ are denoted by $n^{+}$ and $\mathrm{n}^{-}$respectively. The complement of a graph $\mathrm{G}$ is denoted by $\overline{\mathrm{G}}$. A graph $G$ is an $r$-regular graph if all its vertices have same degree equal to $r$. The line graph of $\mathrm{G}$, denoted by $\mathrm{L}(\mathrm{G})$ is a graph whose vertex set has one-to-one correspondence with the edge set of $G$ and two vertices are adjacent in $L(G)$ if the corresponding edges are adjacent in $G$. For $k=1,2, \ldots$, the $k$-th iterated line graph of $G$ is defined as $L^{k}(G)=L\left(L^{k-1}(G)\right)$, where $L^{0}(G)=G$ and $L^{1}(G)=L(G)[10]$. Let $K_{n}$ be the complete graph of order $n$ and $K_{n_{1}, n_{2}, \ldots, n_{k}}$ be the complete multipartite graph of order $n=\sum_{j=1}^{k} n_{j}$.

If $\theta_{1} \geq \theta_{2} \geq \cdots \geq \theta_{\mathrm{n}}$ be the A-eigenvalues of $\mathrm{G}$, then the energy or $A$-energy is defined as

$$
E(G)=\sum_{j=1}^{n}\left|\theta_{j}\right|=2 \sum_{j=1}^{n^{+}} \theta_{j}=-2 \sum_{j=1}^{n^{-}} \theta_{n-j+1} .
$$


Several researchers have introduced many graph operations such as complement, disjoint union, join, graph products etc. The graph products Cartesian product, tensor product and strong product are known as the standard graph products and have been well studied in the graph theory. The energy of a graph introduced in 1978 [8] in connection with molecular chemistry and gained its own importance in the spectral graph theory.

Two graphs $G_{1}$ and $G_{2}$ of same order are said to be equienergetic or $A$ equienergetic if $E\left(G_{1}\right)=E\left(G_{2}\right)$. Similar to A-energy, the Seidel energy or $S$-energy $E_{S}(G)[9]$ of a graph $G$ is defined as the sum of the absolute values of the eigenvalues of Seidel matrix $S$. Two graphs $G_{1}$ and $G_{2}$ of same order are said to be Seidel equienergetic or $S$-equienergetic if $E_{S}\left(G_{1}\right)=E_{S}\left(G_{2}\right)$. Numerous results dealing with the non-cospectral, A-equienergetic graphs have been appeared in the literature. Balakrishnan [2] and Stevanović [23] constructed A-equienergetic graphs using tensor product. Ramane and Walikar [20] and Liu and Liu [12] constructed A-equienergetic graphs by join of two graphs. Bonifácio et al. [3] and Ramane et al. [17] obtained some class of Aequienergetic graphs through Cartesian product, tensor product and strong product. Ramane et al. [21] obtained non-cospectral A-equienergetic iterated line graphs from regular graphs. For other results on A-equienergetic and $\mathrm{S}$ equienergetic graphs one can see $[1,4,7,11,12,13,14,15,18,22,24]$. For other notation, terminology and results related to the spectra of graphs we follow [6]. One of the interesting and difficult problem in the study of energy of a graph in spectral graph theory is to find non-isomorphic graphs of same order with same energy. So for in the literature the linear relations on energies of two non isomorphic graphs are not well studied except A-equienergetic or S-equienergetic graphs. This motivates to find some class of graphs which satisfies the linear relations on energies of different graphs.

This article is organized as follows. In section 2, basic definitions, known results on A-eigenvalues of graph products, A-energy of a graph, S-eigenvalues and $S$-energy of a graph are presented. In section 3 , certain class of $A$-equienergetic graphs are constructed and obtained some linear relations on the A-energies. In section 4 , some class of the S-equienergetic graphs are constructed and obtained some linear relations on the $\mathrm{S}$-energies.

\section{Preliminaries}

In this section, we shall list some known results which are needed in the next two sections. 
Theorem 1 [6] Let $\mathrm{G}$ be an $\mathrm{r}$-regular graph of order $\mathrm{n}$ with the A-eigenvalues $\theta_{1}=r, \theta_{2}, \ldots, \theta_{n}$. Then the $A$-eigenvalues of $\bar{G}$ are $n-r-1,-\theta_{2}-1, \ldots,-\theta_{n}-$ 1.

Theorem 2 [6] Let $\mathrm{G}$ be an $\mathrm{r}$-regular graph on $\mathrm{n}$ vertices and $\mathrm{m}$ edges with the A-eigenvalues $\theta_{1}=r, \theta_{2}, \ldots, \theta_{n}$. Then the $A$-eigenvalues of $L(G)$ are $\theta_{i}+r-2$, $\mathrm{i}=1,2, \ldots, \mathrm{n}$ and $-2(\mathrm{~m}-\mathrm{n}$ times $)$.

Theorem 3 [5] Let $\mathrm{G}$ is an $\mathrm{r}$-regular graph of order $\mathrm{n}$ with the A-eigenvalues $\theta_{1}=r, \theta_{2}, \ldots, \theta_{n}$. Then the eigenvalues of $\mathrm{S}$ are $\mathrm{n}-2 \mathrm{r}-1,-1-2 \theta_{2}, \ldots,-1-$ $2 \theta_{n}$.

Theorem 4 [16] Let $\mathrm{G}_{1}$ and $\mathrm{G}_{2}$ be two r-regular graphs of same order $\mathrm{n}$, $\mathrm{r} \geq 3$. Then for $\mathrm{k} \geq 2, \mathrm{~L}^{\mathrm{k}}\left(\mathrm{G}_{1}\right)$ and $\mathrm{L}^{\mathrm{k}}\left(\mathrm{G}_{2}\right)$ are $\mathrm{S}$-equienergetic.

The Cartesian product of two graphs $G_{1}$ and $G_{2}$ is the graph $G_{1} \square G_{2}$ with vertex set $V\left(G_{1}\right) \times V\left(G_{2}\right)$, in which the vertices $\left(u_{1}, u_{2}\right)$ and $\left(v_{1}, v_{2}\right)$ are adjacent if either $u_{1}$ is adjacent to $v_{1}$ in $G_{1}$ and $u_{2}$ is equal to $v_{2}$ or $u_{1}$ is equal to $v_{1}$ and $u_{2}$ is adjacent to $v_{2}$ in $G_{2}$.

The tensor product of two graphs $G_{1}$ and $G_{2}$ is the graph $G_{1} \otimes G_{2}$ with vertex set $V\left(G_{1}\right) \times V\left(G_{2}\right)$, in which the vertices $\left(u_{1}, u_{2}\right)$ and $\left(v_{1}, v_{2}\right)$ are adjacent if $u_{1}$ is adjacent to $v_{1}$ in $G_{1}$ and $u_{2}$ is adjacent to $v_{2}$ in $G_{2}$.

The strong product of two graphs $G_{1}$ and $G_{2}$ is the graph $G_{1} \otimes G_{2}$ with vertex set $V\left(G_{1}\right) \times V\left(G_{2}\right)$, in which the vertices $\left(u_{1}, u_{2}\right)$ and $\left(v_{1}, v_{2}\right)$ are adjacent whenever $u_{1}$ and $v_{1}$ are equal or adjacent in $G_{1}$, and $u_{2}$ and $v_{2}$ are equal or adjacent in $\mathrm{G}_{2}$. If $\mathrm{G}_{1}$ and $\mathrm{G}_{2}$ are two regular graphs then $\mathrm{G}_{1} \square \mathrm{G}_{2}, \mathrm{G}_{1} \otimes \mathrm{G}_{2}$ and $\mathrm{G}_{1} \otimes \mathrm{G}_{2}$ are also regular graphs.

Lemma 5 [5] If $\mu_{1}, \mu_{2}, \ldots, \mu_{n}$ are the A-eigenvalues of a graph $\mathrm{G}_{1}$ and $\sigma_{1}, \sigma_{2}$, $\ldots, \sigma_{m}$ are the $A$-eigenvalues of a graph $\mathrm{G}_{2}$ then

(i) the A-eigenvalues of $\mathrm{G}_{1} \square \mathrm{G}_{2}$ are $\mu_{i}+\sigma_{j}, i=1,2, \ldots, n ; j=1,2, \ldots, m$,

(ii) the A-eigenvalues of $\mathrm{G}_{1} \otimes \mathrm{G}_{2}$ are $\mu_{i} \sigma_{j}, i=1,2, \ldots, n ; j=1,2, \ldots, \mathrm{m}$,

(iii) the A-eigenvalues of $\mathrm{G}_{1} \otimes \mathrm{G}_{2}$ are $\mu_{i} \sigma_{j}+\mu_{i}+\sigma_{j}, i=1,2, \ldots, n ; j=$ $1,2, \ldots, \mathrm{m}$.

Lemma 6 [17] The A-spectrum of the line graph of a complete bipartite graph $\mathrm{K}_{\mathrm{p}, \mathrm{q}}$, where $\mathrm{p}, \mathrm{q} \geq 2$ is

$$
\operatorname{Spec}\left(L\left(K_{p, q}\right)\right)=\left(\begin{array}{cccc}
p+q-2 & p-2 & q-2 & -2 \\
1 & q-1 & p-1 & (p-1)(q-1)
\end{array}\right) .
$$




\section{A-equienergetic graphs and linear relations on $A-$ energies of certain class of graphs}

\subsection{A-equienergetic graphs}

Theorem 7 Let $\mathrm{G}_{1}$ and $\mathrm{G}_{2}$ be two r-regular $\mathrm{A}$-equienergetic graphs of order

$\mathrm{n}$. Then for $\mathrm{p} \geq \mathrm{r}, \mathrm{E}(\mathrm{G}_{1} \square \mathrm{K} \underbrace{\mathrm{p}, \mathrm{p}, \ldots, \mathrm{p}}_{\mathrm{k} \text { times }})=\mathrm{E}(\mathrm{G}_{2} \square \mathrm{K} \underbrace{\mathrm{p}, \mathrm{p}, \ldots, \mathrm{p}}_{\mathrm{k} \text { times }})$.

Proof. We have, $\operatorname{Spec}(\underbrace{K_{1}^{p, p, \ldots, p}}_{k \text { times }})=\left(\begin{array}{ccc}p(k-1) & 0 & -p \\ 1 & k(p-1) & k-1\end{array}\right)$.

Let $\operatorname{Spec}\left(G_{1}\right)=\left(\begin{array}{cccc}r & \theta_{2} & \ldots & \theta_{k} \\ 1 & m_{2} & \ldots & m_{k}\end{array}\right)$, where $1+\sum_{j=2}^{k} m_{j}=n$.

By (i) of Lemma 5,

$$
\begin{aligned}
& \operatorname{Spec}\left(G_{1} \square K_{K_{\text {times }}^{p, p, \ldots, p}}^{p, \ldots}\right)=\left(\begin{array}{ccccc}
r+p(k-1) & r & r-p & \theta_{2}+p(k-1) & \ldots \\
1 & k(p-1) & k-1 & m_{2} & \ldots
\end{array}\right. \\
& \left.\begin{array}{ccccccc}
\theta_{k}+p(k-1) & \theta_{2} & \cdots & \theta_{k} & \theta_{2}-p & \cdots & \theta_{k}-p \\
m_{k} & k m_{2}(p-1) & \cdots & k m_{k}(p-1) & m_{2}(k-1) & \cdots & m_{k}(k-1)
\end{array}\right) .
\end{aligned}
$$

Therefore

$$
\begin{aligned}
E(G_{1} \square \underbrace{p, p, \ldots, p}_{k \text { times }}) \\
=\quad|r+p(k-1)|+|r| k(p-1)+|r-p|(k-1)+\sum_{i=2}^{k} m_{i}\left|\theta_{i}+p(k-1)\right| \\
\quad+\sum_{i=2}^{k} k m_{i}(p-1)\left|\theta_{i}\right|+\sum_{i=2}^{k} m_{i}(k-1)\left|\theta_{i}-p\right| \\
=\quad r+p(k-1)+k r(p-1)+(p-r)(k-1)+\sum_{i=2}^{k} m_{i}\left(\theta_{i}+p(k-1)\right) \\
\quad+k(p-1) \sum_{i=2}^{k} m_{i}\left|\theta_{i}\right|+\sum_{i=2}^{k} m_{i}(k-1)\left(p-\theta_{i}\right) \\
\quad \operatorname{since~} \theta_{i}+p(k-1) \geq-r+p \geq 0 \text { and } \theta_{i}-p \leq r-p \leq 0 \\
=\quad 2 n p(k-1)+k(p-1) E\left(G_{1}\right) .
\end{aligned}
$$


Since $G_{2}$ is also an $r$-regular graph of order $n$, we have

$$
\mathrm{E}\left(\mathrm{G}_{2} \square \mathrm{K}_{\underbrace{\mathrm{p}, \mathrm{p}, \ldots, \mathrm{p}}_{\mathrm{k} \text { times }}}\right)=2 \mathrm{np}(\mathrm{k}-1)+\mathrm{k}(\mathrm{p}-1) \mathrm{E}\left(\mathrm{G}_{2}\right) .
$$

If $G_{1}$ and $G_{2}$ are A-equienergetic then $E\left(G_{1} \square K_{k_{\text {times }}}^{p, p, \ldots, p}\right)=E\left(G_{2} \square K_{k}^{p, p, \ldots, p}\right)$

which completes the proof.

Remark 8 Recently in [19] Ramane et al. proved that $\mathrm{E}\left(\mathrm{G}_{1} \square \mathrm{K}_{\mathrm{p}, \mathrm{p}}\right)=\mathrm{E}\left(\mathrm{G}_{2} \square \mathrm{K}_{\mathrm{p}, \mathrm{p}}\right)$. It is noted that this result becomes particular case of Theorem 7.

Theorem 9 If $\mathrm{p} \geq \mathrm{n} \geq 2$ and $\mathrm{k} \geq 2$ then

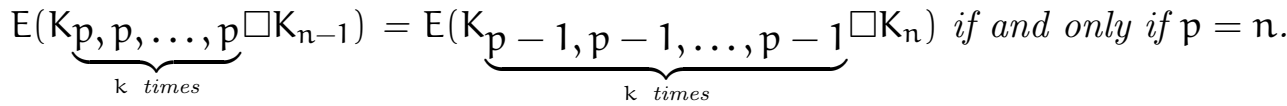

Proof. We have

$$
\operatorname{Spec}(\underbrace{\mathrm{p}, p, \ldots, p}_{k \text { times }})=\left(\begin{array}{ccc}
p(k-1) & 0 & -p \\
1 & k(p-1) & k-1
\end{array}\right)
$$

and

$$
\operatorname{Spec}\left(K_{n-1}\right)=\left(\begin{array}{cc}
n-2 & -1 \\
1 & n-2
\end{array}\right)
$$

By (i) of Lemma 5 ,

$$
\begin{aligned}
& \operatorname{Spec}\left(K_{k \text { times }}^{p, p, \ldots, p} \square K_{n-1}\right)=\left(\begin{array}{ccc}
p k-p+n-2 & p k-p-1 & n-2 \\
1 & n-2 & k(p-1)
\end{array}\right. \\
& \left.\begin{array}{ccc}
-1 & n-p-2 & -p-1 \\
k(n-2)(p-1) & k-1 & (k-1)(n-2)
\end{array}\right) .
\end{aligned}
$$

If $p \geq n \geq 2$ then $p k-p+n-2, p k-p-1$ and $n-2$ are only the positive A-eigenvalues of $K_{k_{\text {times }}, p, \ldots, p} \square K_{n-1}$. Therefore from definition of A-energy, we get

$$
\begin{aligned}
& \mathrm{E}\left(\mathrm{K}_{\mathrm{k} \text { times }}^{\mathrm{p}, \mathrm{p}, \ldots, \mathrm{p}} \square \mathrm{K}_{n-1}\right) \\
& \quad=2[\mathrm{pk}-\mathrm{p}+\mathrm{n}-2+(\mathrm{n}-2)(\mathrm{pk}-\mathrm{p}-1)+\mathrm{k}(\mathrm{n}-2)(\mathrm{p}-1)] \\
& \quad=2[2 n p k-3 p k-n p+p-n k+2 k] .
\end{aligned}
$$


Next, we have

$$
\operatorname{Spec}(\underbrace{p-1, p-1, \ldots, p-1}_{k \text { times }})=\left(\begin{array}{ccc}
(p-1)(k-1) & 0 & -(p-1) \\
1 & k(p-2) & k-1
\end{array}\right)
$$

and

$$
\operatorname{Spec}\left(K_{n}\right)=\left(\begin{array}{cc}
n-1 & -1 \\
1 & n-1
\end{array}\right)
$$

By (i) of Lemma 5,

$$
\begin{aligned}
& \operatorname{Spec}\left(K_{K_{\text {times }}}^{p-1, p-1, \ldots, p-1} \square K_{n}\right) \\
& =\left(\begin{array}{ccc}
p k-p+n-k & p k-p-k & n-1 \\
1 & n-1 & k(p-2)
\end{array}\right. \\
& \left.\begin{array}{ccc}
-1 & n-p & -p \\
k(n-1)(p-2) & k-1 & (k-1)(n-1)
\end{array}\right) .
\end{aligned}
$$

If $p \geq n \geq 2$ then $p k-p+n-k, p k-p-k$ and $n-1$ are only the positive

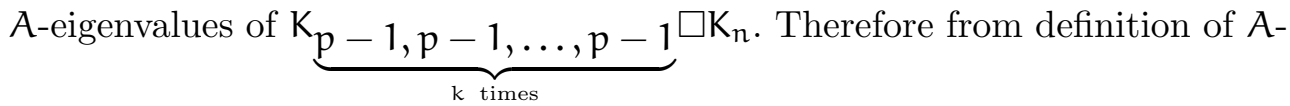
energy, we get

$$
\begin{aligned}
& \mathrm{E}(\underbrace{\mathrm{K}-1, p-1, \ldots, p-1}_{k \text { times }} \square K_{n}) \\
& \quad=2[p k-p-k+n+(n-1)(p k-p-k)+k(n-1)(p-2)] \\
& =2[2 n p k-3 n k-n p+n-p k+2 k] .
\end{aligned}
$$

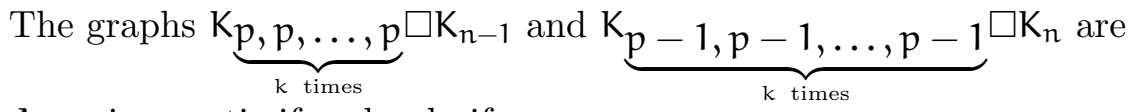
A-equienergetic if and only if

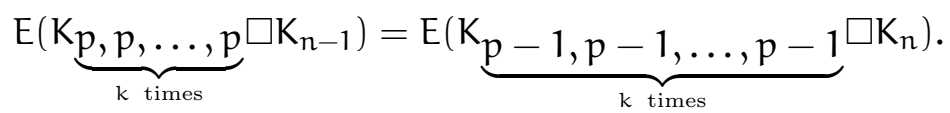

That is, $2[2 n p k-3 p k-n p+p-n k+2 k]=2[2 n p k-3 n k-n p+n-p k+2 k]$ or $p+2 n k=n+2 p k$, which implies $(n-p)(2 k-1)=0$. Since $k$ is positive integer, we get $p=n$. This completes the proof. 
Theorem 10 Let $\mathrm{G}$ be the Petersen graph and $\mathrm{p}, \mathrm{q} \geq 4$. Then $\mathrm{E}\left(\mathrm{G} \square \overline{\mathrm{L}\left(\mathrm{K}_{\mathrm{p}, \mathrm{q}}\right)}\right)=$ $\mathrm{E}\left(\overline{\mathrm{G}} \square \mathrm{L}\left(\mathrm{K}_{\mathrm{p}, \mathrm{q}}\right)\right)$.

Proof. The A-spectrum of Petersen graph is

$$
\operatorname{Spec}(\mathrm{G})=\left(\begin{array}{ccc}
3 & 1 & -2 \\
1 & 5 & 4
\end{array}\right)
$$

From Lemma 6 and Theorem 1, A-spectrum of $\overline{\mathrm{L}\left(\mathrm{K}_{\mathrm{p}, \mathrm{q}}\right)}$ is

$$
\operatorname{Spec}\left(\overline{L\left(K_{p, q}\right)}\right)=\left(\begin{array}{cccc}
p q-p-q+1 & 1-p & 1-q & 1 \\
1 & q-1 & p-1 & (p-1)(q-1)
\end{array}\right) .
$$

Now by (i) of Lemma 5 , A-spectrum of $G \square \overline{\mathrm{L}\left(\mathrm{K}_{\mathrm{p}, \mathrm{q}}\right)}$ is

$$
\begin{aligned}
& \left(\begin{array}{cccccc}
p q-p-q+4 & 4-p & 4-q & 4 & p q-p-q+2 & 2-p \\
1 & q-1 & p-1 & p q-p-q+1 & 5 & 5 q-5
\end{array}\right. \\
& \begin{array}{ccccc}
2-q & 2 & p q-p-q-1 & -1-p & -1-q \\
5 p-5 & 5 p q-5 p-5 q+5 & 4 & 4 q-4 & 4 p-4
\end{array} \\
& 4 p q-4 p-4 q+4) \text {. }
\end{aligned}
$$

If $p, q \geq 4$ then $p q-p-q+4,4, p q-p-q+2,2$ and $p q-p-q-1$ are only the positive A-eigenvalues of $\mathrm{G} \square \overline{\mathrm{L}\left(\mathrm{K}_{p, q}\right)}$. Therefore from definition of A-energy

$$
\begin{aligned}
E\left(G \square \overline{\left(K_{p, q}\right)}\right) & =2[p q-p-q+4+4(p q-p-q-1)+5(p q-p-q+2) \\
& +2(5 p q-5 p-5 q+5)+4(p q-p-q+1)] \\
& =48(p-1)(q-1)
\end{aligned}
$$

Now

$$
\operatorname{Spec}(\overline{\mathrm{G}})=\left(\begin{array}{ccc}
6 & 1 & -2 \\
1 & 4 & 5
\end{array}\right) .
$$

By using (i) of Lemma 5 and Lemma $6, \overline{\mathrm{G}} \square \mathrm{L}\left(\mathrm{K}_{\mathrm{p}, \mathrm{q}}\right)$ has A-spectrum,

$$
\left(\begin{array}{ccccccc}
p+q+4 & p+4 & q+4 & 4 & p+q-4 & p-4 & q-4 \\
1 & q-1 & p-1 & p q-p-q+1 & 5 & 5 q-5 & 5 p-5 \\
-4 & & p+q-1 & p-1 & q-1 & -1 \\
5 p q-5 p-5 q+5 & 4 & 4 q-4 & 4 p-4 & 4 p q-4 p-4 q+4
\end{array}\right)
$$


If $p, q \geq 4$ then -4 and -1 are only the negative $A$-eigenvalues of $\bar{G} \square L\left(K_{p, q}\right)$. Therefore from definition of A-energy

$$
\begin{aligned}
\mathrm{E}\left(\overline{\mathrm{G}} \square \mathrm{L}\left(\mathrm{K}_{\mathrm{p}, \mathrm{q}}\right)\right) & =2[4(5 p q-5 p-5 q+5)+4 p q-4 p-4 q+4] \\
& =48(p-1)(q-1)
\end{aligned}
$$

which completes the proof.

Recently in [17] Ramane et al. proved that $\mathrm{E}\left(\mathrm{L}\left(\mathrm{K}_{\mathrm{p}, q}\right)\right)=\mathrm{E}\left(\overline{\mathrm{L}\left(\mathrm{K}_{\mathrm{p}, q}\right)}\right)$. In the following A-equienergetic graphs with the help of these graphs are given.

Theorem 11 If $\mathrm{p}, \mathrm{q} \geq 5$ then $\mathrm{E}\left(\mathrm{L}\left(\mathrm{K}_{\mathrm{p}, \mathrm{q}}\right) \square \mathrm{L}\left(\mathrm{K}_{4}\right)\right)=\mathrm{E}\left(\overline{\mathrm{L}\left(\mathrm{K}_{\mathrm{p}, \mathrm{q}}\right)} \square \mathrm{L}\left(\mathrm{K}_{4}\right)\right)$.

Proof. The A-spectrum of $K_{4}$ is

$$
\operatorname{Spec}\left(\mathrm{K}_{4}\right)=\left(\begin{array}{cc}
3 & -1 \\
1 & 3
\end{array}\right)
$$

From Theorem 2,

$$
\operatorname{Spec}\left(L\left(K_{4}\right)\right)=\left(\begin{array}{ccc}
4 & 0 & -2 \\
1 & 3 & 2
\end{array}\right)
$$

By using (i) of Lemma 5 and Lemma 6, $\mathrm{L}\left(\mathrm{K}_{\mathrm{p}, \mathrm{q}}\right) \square \mathrm{L}\left(\mathrm{K}_{4}\right)$ has A-spectrum

$$
\left(\begin{array}{cccccccc}
p+q+2 & p+q-2 & p+q-4 & p+2 & p-2 & p-4 & q+2 & q-2 \\
1 & 3 & 2 & q-1 & 3 q-3 & 2 q-2 & p-1 & 3 p-3 \\
& q-4 & -4 & 2 & -2 \\
2 p-2 & 2 p q-2 p-2 q+2 & p q-p-q+1 & 3 p q-3 p-3 q+3
\end{array}\right) .
$$

If $p, q \geq 5$ then -4 and -2 are only the negative $A$-eigenvalues of $L\left(K_{p, q}\right) \square L\left(K_{4}\right)$. Therefore from definition of A-energy

$$
\begin{aligned}
\mathrm{E}\left(\mathrm{L}\left(\mathrm{K}_{\mathrm{p}, \mathrm{q}}\right) \square \mathrm{L}\left(\mathrm{K}_{4}\right)\right) & =2[4(2 p q-2 p-2 q+2)+2(3 p q-3 p-3 q+3)] \\
& =28(p-1)(q-1) .
\end{aligned}
$$

By using Theorem 1, (i) of Lemma 5 and Lemma $6, \overline{\mathrm{L}\left(\mathrm{K}_{\mathrm{p}, \mathrm{q}}\right)} \square \mathrm{L}\left(\mathrm{K}_{4}\right)$ has Aspectrum,

$$
\begin{aligned}
& \left(\begin{array}{cccccc}
p q-p-q+5 & p q-p-q+1 & p q-p-q-1 & 5-p & 1-p & -1-p \\
1 & 3 & 2 & q-1 & 3 q-3 & 2 q-2
\end{array}\right. \\
& \begin{array}{lllll}
5-q & 1-q & -1-q & 5 & 1
\end{array} \\
& p-1 \quad 3 p-3 \quad 2 p-2 \quad p q-p-q+1 \quad 3 p q-3 p-3 q+3 \\
& 2 p q-2 p-2 q+2) \text {. }
\end{aligned}
$$


If $p, q \geq 5$ then $p q-p-q+5, p q-p-q+1, p q-p-q-1,5$ and 1 are only the positive A-eigenvalues of $\overline{\mathrm{L}\left(\mathrm{K}_{\mathrm{p}, q}\right)} \square \mathrm{L}\left(\mathrm{K}_{4}\right)$. Therefore from definition of A-energy

$$
\begin{aligned}
\mathrm{E}\left(\overline{\mathrm{L}\left(\mathrm{K}_{\mathrm{p}, \mathrm{q}}\right)} \square \mathrm{L}\left(\mathrm{K}_{4}\right)\right) & =2[\mathrm{pq}-\mathrm{p}-\mathrm{q}+5+3(\mathrm{pq}-\mathrm{p}-\mathrm{q}+1)+2(\mathrm{pq}-\mathrm{p}-\mathrm{q}-1) \\
& +5(\mathrm{pq}-\mathrm{p}-\mathrm{q}+1)+3 \mathrm{pq}-3 \mathrm{p}-3 \mathrm{q}+3] \\
& =28(\mathrm{p}-1)(\mathrm{q}-1)
\end{aligned}
$$

which completes the proof.

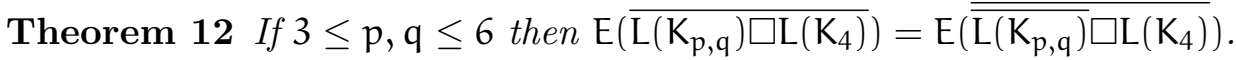

Proof. We have

$$
\operatorname{Spec}\left(L\left(K_{4}\right)\right)=\left(\begin{array}{ccc}
4 & 0 & -2 \\
1 & 3 & 2
\end{array}\right)
$$

By using (i) of Lemma 5, Lemma 6 and Theorem 1, $\overline{\mathrm{L}\left(\mathrm{K}_{\mathrm{p}, \mathrm{q}}\right) \square \mathrm{L}\left(\mathrm{K}_{4}\right)}$ has Aspectrum

$$
\left(\begin{array}{ccccc}
6 p q-p-q-3 & -1-p-q+2 & -1-p-q+4 & -1-p-2 & -1-p+2 \\
1 & 3 & 2 & q-1 & 3 q-3 \\
-1-p+4 & -1-q-2 & -1-q+2 & -1-q+4 & -3 \\
2 q-2 & p-1 & 3 p-3 & 2 p-2 & p q-p-q+1 \\
& 3 p q-3 p-3 q+3 & 2 p q-2 p-2 q+2
\end{array}\right) .
$$

If $p, q \geq 3$ then $6 p q-p-q-3,1$ and 3 are only the positive $A$-eigenvalues of $\overline{L\left(K_{p, q}\right) \square L\left(K_{4}\right)}$. Therefore from definition of A-energy, we have $\mathrm{E}\left(\overline{\mathrm{L}\left(\mathrm{K}_{\mathrm{p}, q}\right) \square \mathrm{L}\left(\mathrm{K}_{4}\right)}\right)$

$$
\begin{aligned}
& =2[6 p q-p-q-3+1(3 p q-3 p-3 q+3)+3(2 p q-2 p-2 q+2)] \\
& =2(15 p q-10 p-10 q+6) .
\end{aligned}
$$

By using (i) of Lemma 5, Lemma 6 and Theorem 1, $\overline{\overline{\mathrm{L}\left(\mathrm{K}_{\mathrm{p}, \mathrm{q}}\right)}} \square \mathrm{L}\left(\mathrm{K}_{4}\right)$ has Aspectrum

$$
\begin{aligned}
& \left(\begin{array}{cccccc}
5 p q+p+q-6 & p+q-p q-2 & p+q-p q & p-6 & p-2 & p \\
1 & 3 & 2 & q-1 & 3 q-3 & 2 q-2
\end{array}\right. \\
& \begin{array}{lllll}
q-6 & q-2 & q & -6 & -2
\end{array} \\
& p-1 \quad 3 p-3 \quad 2 p-2 \quad p q-p-q+1 \quad 3 p q-3 p-3 q+3 \\
& 2 p q-2 p-2 q+2) \text {. }
\end{aligned}
$$


If $3 \leq p, q \leq 6$ then $5 p q+p+q-6, p-2, p, q-2$ and $q$ are only the positive A-eigenvalues of $\overline{\overline{L\left(K_{p, q}\right)}} \square \mathrm{L}\left(K_{4}\right)$. Therefore from definition of A-energy

$$
\begin{aligned}
& \mathrm{E}\left(\overline{\overline{\mathrm{L}\left(\mathrm{K}_{\mathrm{p}, \mathrm{q}}\right)}} \square \mathrm{L}\left(\mathrm{K}_{4}\right)\right)=2[5 \mathrm{pq}+\mathrm{p}+\mathrm{q}-6+(\mathrm{p}-2)(3 \mathrm{q}-3)+\mathrm{p}(2 \mathrm{q}-2) \\
& +(q-2)(3 p-3)+q(2 p-2)] \\
& =2(15 p q-10 p-10 q+6)
\end{aligned}
$$

which completes the proof.

Theorem 13 If $\mathrm{p}, \mathrm{q} \geq 3$ then $\mathrm{E}\left(\mathrm{L}\left(\mathrm{K}_{\mathrm{p}, \mathrm{q}}\right) \square \overline{\mathrm{L}\left(\mathrm{K}_{4}\right)}\right)=\mathrm{E}\left(\overline{\mathrm{L}\left(\mathrm{K}_{\mathrm{p}, \mathrm{q}}\right)} \square \overline{\mathrm{L}\left(\mathrm{K}_{4}\right)}\right)$.

Proof. We have

$$
\operatorname{Spec}\left(L\left(K_{4}\right)\right)=\left(\begin{array}{ccc}
4 & 0 & -2 \\
1 & 3 & 2
\end{array}\right)
$$

and from Theorem 1

$$
\operatorname{Spec}\left(\overline{\mathrm{L}\left(\mathrm{K}_{4}\right)}\right)=\left(\begin{array}{cc}
1 & -1 \\
3 & 3
\end{array}\right)
$$

By using (i) of Lemma 5 and Lemma 6, $\mathrm{L}\left(\mathrm{K}_{\mathrm{p}, \mathrm{q}}\right) \square \overline{\mathrm{L}\left(\mathrm{K}_{4}\right)}$ has A-spectrum

$$
\begin{aligned}
& \left(\begin{array}{cccccc}
p+q-1 & p+q-3 & p-1 & p-3 & q-1 & q-3 \\
3 & 3 & 3 q-3 & 3 q-3 & 3 p-3 & 3 p-3
\end{array}\right. \\
& \left.\begin{array}{cc}
-1 & -3 \\
3 p q-3 p-3 q+3 & 3 p q-3 p-3 q+3
\end{array}\right) \text {. }
\end{aligned}
$$

If $p, q \geq 3$ then -1 and -3 are only the negative $A$-eigenvalues of $L\left(K_{p, q}\right) \square \overline{L\left(K_{4}\right)}$. Therefore from definition of A-energy

$$
\begin{aligned}
\mathrm{E}\left(\mathrm{L}\left(\mathrm{K}_{\mathrm{p}, \mathrm{q}}\right) \square \overline{\mathrm{L}\left(\mathrm{K}_{4}\right)}\right) & =2[3(\mathrm{p}-1)(\mathrm{q}-1)+9(\mathrm{p}-1)(\mathrm{q}-1)] \\
& =24(\mathrm{p}-1)(\mathrm{q}-1) .
\end{aligned}
$$

By using Lemma 6, Theorem 1 and (i) of Lemma $5, \overline{\mathrm{L}\left(\mathrm{K}_{\mathrm{p}, \mathrm{q}}\right)} \square \overline{\mathrm{L}\left(\mathrm{K}_{4}\right)}$ has Aspectrum

$$
\begin{gathered}
\left(\begin{array}{cccccc}
p q-p-q+2 & p q-p-q & 2-p & -p & 2-q & -q \\
3 & 3 & 3 q-3 & 3 q-3 & 3 p-3 & 3 p-3 \\
2 & 0 & \\
3 p q-3 p-3 q+3 & 3 p q-3 p-3 q+3
\end{array}\right) .
\end{gathered}
$$


If $p, q \geq 3$ then $p q-p-q+2, p q-p-q$ and 2 are only the positive A-eigenvalues of $\overline{\mathrm{L}\left(\mathrm{K}_{p, q}\right)} \square \overline{\mathrm{L}\left(\mathrm{K}_{4}\right)}$. Therefore from definition of A-energy

$$
\begin{aligned}
\mathrm{E}\left(\overline{\mathrm{L}\left(\mathrm{K}_{\mathrm{p}, \mathrm{q}}\right)} \square \overline{\mathrm{L}\left(\mathrm{K}_{4}\right)}\right) & =2[3(\mathrm{pq}-\mathrm{p}-\mathrm{q}+2)+3(\mathrm{pq}-\mathrm{p}-\mathrm{q}) \\
& +2(3 p q-3 p-3 \mathrm{q}+3)] \\
& =24(\mathrm{p}-1)(\mathrm{q}-1)
\end{aligned}
$$

which completes the proof.

Theorem 14 If $\mathrm{p}, \mathrm{q} \geq 4$ then $\mathrm{E}\left(\overline{\mathrm{L}\left(\mathrm{K}_{\mathrm{p}, \mathrm{q}}\right)} \square \mathrm{C}_{6}\right)=\mathrm{E}\left(\mathrm{L}\left(\mathrm{K}_{\mathrm{p}, \mathrm{q}}\right) \square \overline{\mathrm{C}_{6}}\right)$ where $\mathrm{C}_{6}$ is the cycle of order 6 .

Proof. We have

$$
\operatorname{Spec}\left(\mathrm{C}_{6}\right)=\left(\begin{array}{cccc}
2 & 1 & -1 & -2 \\
1 & 2 & 2 & 1
\end{array}\right)
$$

By using Lemma 6, Theorem 1 and (i) of Lemma 5, A-spectrum of $\overline{\mathrm{L}\left(\mathrm{K}_{\mathrm{p}, \mathrm{q}}\right)} \square \mathrm{C}_{6}$ is

$$
\begin{aligned}
& \left(\begin{array}{ccccc}
p q-p-q+3 & p q-p-q+2 & p q-p-q & p q-p-q-1 & 3-p \\
1 & 2 & 2 & 1 & q-1
\end{array}\right. \\
& \begin{array}{llllllll}
2-\mathrm{p} & -\mathrm{p} & -1-\mathrm{p} & 3-\mathrm{q} & 2-\mathrm{q} & -\mathrm{q} & -1-\mathrm{q} & 3
\end{array} \\
& 2 q-2 \quad 2 q-2 \quad q-1 \quad p-1 \quad 2 p-2 \quad 2 p-2 \quad p-1 \quad p q-p-q+1 \\
& \left.\begin{array}{ccc}
2 & 0 & -1 \\
2 p q-2 p-2 q+2 & 2 p q-2 p-2 q+2 & p q-p-q+1
\end{array}\right) \text {. }
\end{aligned}
$$

If $p, q \geq 4$ then $p q-p-q+3, p q-p-q+2, p q-p-q, p q-p-q-1,3$ and 2 are only the positive $A$-eigenvalues of $\overline{L\left(K_{p, q}\right)} \square C_{6}$. Therefore from definition of A-energy

$$
\begin{aligned}
& E\left(\overline{L\left(K_{p, q}\right)} \square C_{6}\right) \\
& \quad=2[p q-p-q+3+2(p q-p-q+2)+2(p q-p-q) \\
& \quad+(p q-p-q-1)+3(p q-p-q+1)+2(2 p q-2 p-2 q+2)] \\
& \quad=26(p-1)(q-1) .
\end{aligned}
$$

Now by Theorem 1

$$
\operatorname{Spec}\left(\overline{\mathrm{C}_{6}}\right)=\left(\begin{array}{cccc}
3 & 1 & 0 & -2 \\
1 & 1 & 2 & 2
\end{array}\right)
$$


By using Lemma 6 and (i) of Lemma $5 \mathrm{~L}\left(\mathrm{~K}_{\mathrm{p}, \mathrm{q}}\right) \square \overline{\mathrm{C}_{6}}$ has A-spectrum

$$
\left.\begin{array}{cccccccc}
p+q+1 & p+q-1 & p+q-2 & p+q-4 & p+1 & p-1 & p-2 & p-4 \\
1 & 1 & 2 & 2 & q-1 & q-1 & 2 q-2 & 2 q-2 \\
q+1 & q-1 & q-2 & q-4 & 1 & -1 \\
p-1 & p-1 & 2 p-2 & 2 p-2 & p q-p-q+1 & p q-p-q+1 \\
& & & & -2 & -4 \\
& & & & & p q-2 p-2 q+2 & 2 p q-2 p-2 q+2
\end{array}\right) .
$$

If $p, q \geq 4$ then $-1,-2$ and -4 are only the negative $A$-eigenvalues of $L\left(K_{p, q}\right) \square \overline{\mathrm{C}_{6}}$. Therefore from definition of A-energy

$$
\begin{aligned}
\mathrm{E}\left(\mathrm{L}\left(\mathrm{K}_{\mathrm{p}, \mathrm{q}}\right) \square \overline{\mathrm{C}_{6}}\right) & =2[\mathrm{pq}-\mathrm{p}-\mathrm{q}+1+2(2 p q-2 p-2 q+2) \\
& +4(2 p q-2 p-2 q+2)] \\
& =26(p-1)(q-1)
\end{aligned}
$$

which completes the proof.

Theorem 15 If $\mathrm{p}, \mathrm{q} \geq 3$ then $\mathrm{E}\left(\mathrm{L}\left(\mathrm{K}_{\mathrm{p}, \mathrm{q}}\right) \square \overline{\mathrm{W}_{5}}\right)=\mathrm{E}\left(\overline{\mathrm{L}\left(\mathrm{K}_{\mathrm{p}, \mathrm{q}}\right)} \square \overline{\mathrm{W}_{5}}\right)$ where $\mathrm{W}_{5}$ is the wheel of order 5 .

Proof. Since A-spectrum of $\overline{W_{5}}$ is

$$
\left(\begin{array}{ccc}
1 & 0 & -1 \\
2 & 1 & 2
\end{array}\right)
$$

Now by (i) of Lemma 5, A-spectrum of $L\left(K_{p, q}\right) \square \overline{W_{5}}$ is

$$
\left(\begin{array}{cccccccc}
p+q-1 & p+q-2 & p+q-3 & p-1 & p-2 & p-3 & q-1 & q-2 \\
2 & 1 & 2 & 2 q-2 & q-1 & 2 q-2 & 2 p-2 & p-1 \\
& q-3 & -1 & & -2 & -3 \\
2 p-2 & 2 p q-2 p-2 q+2 & p q-p-q+1 & 2 p q-2 p-2 q+2
\end{array}\right) .
$$

If $p, q \geq 3$ then $-1,-2$ and -3 are only the negative $A$-eigenvalues of $L\left(K_{p, q}\right) \square \overline{W_{5}}$. Therefore from definition of A-energy

$$
\begin{aligned}
E\left(L\left(K_{p, q}\right) \square \overline{W_{5}}\right) & =2[2 p q-2 p-2 q+2+2(p q-p-q+1) \\
& +3(2 p q-2 p-2 q+2)] \\
& =20(p-1)(q-1) .
\end{aligned}
$$


By using Lemma 6, Theorem 1 and (i) of Lemma 5 , A-spectrum of $\overline{\mathrm{L}\left(\mathrm{K}_{\mathrm{p}, q}\right)} \square \overline{\mathrm{W}_{5}}$ is

$$
\begin{aligned}
& \left(\begin{array}{ccccccc}
p q-p-q+2 & p q-p-q+1 & p q-p-q & 2-p & 1-p & -p & 2-q \\
2 & 1 & 2 & 2 q-2 & q-1 & 2 q-2 & 2 p-2
\end{array}\right. \\
& \left.\begin{array}{ccccc}
1-q & -q & 2 & 1 & 0 \\
p-1 & 2 p-2 & 2 p q-2 p-2 q+2 & p q-p-q+1 & 2 p q-2 p-2 q+2
\end{array}\right) .
\end{aligned}
$$

If $p, q \geq 3$ then $p q-p-q+2, p q-p-q+1, p q-p-q, 2$ and 1 are only the positive $A$-eigenvalues of $\bar{L}\left(K_{p, q}\right) \square \overline{W_{5}}$. Therefore from definition of A-energy

$$
\begin{aligned}
E\left(\overline{\mathrm{L}\left(\mathrm{K}_{\mathrm{p}, q}\right)} \square \overline{W_{5}}\right) & =2[2(p q-p-q+2)+p q-p-q+1+2(p q-p-q) \\
& +2(2 p q-2 p-2 q+2)+(p q-p-q+1)] \\
& =20(p-1)(q-1)
\end{aligned}
$$

which completes the proof.

Theorem 16 If $n \geq 3$ then $\mathrm{E}\left(\overline{\mathrm{K}_{n, n} \otimes \mathrm{K}_{n-1}}\right)=\mathrm{E}\left(\overline{\mathrm{K}_{n-1, n-1} \otimes \mathrm{K}_{n}}\right)$.

Proof. We have

$$
\operatorname{Spec}\left(K_{n, n}\right)=\left(\begin{array}{ccc}
n & 0 & -n \\
1 & 2 n-2 & 1
\end{array}\right)
$$

and

$$
\operatorname{Spec}\left(K_{n-1}\right)=\left(\begin{array}{cc}
n-2 & -1 \\
1 & n-2
\end{array}\right)
$$

By using (ii) of Lemma 5 and Theorem 1,

$$
\operatorname{Spec}\left(\overline{K_{n, n} \otimes K_{n-1}}\right)=\left(\begin{array}{ccccc}
n^{2}-1 & -1-n & -1 & n(n-2)-1 & n-1 \\
1 & n-2 & (n-1)(2 n-2) & 1 & n-2
\end{array}\right) .
$$

If $n \geq 3$ then $n^{2}-1, n(n-2)-1$ and $n-1$ are only the positive $A$-eigenvalues of $\overline{K_{n, n} \otimes K_{n-1}}$ and from definition of A-energy

$$
\begin{aligned}
E\left(\overline{K_{n, n} \otimes K_{n-1}}\right) & =2\left[n^{2}-1-1+n(n-2)+(n-1)(n-2)\right] \\
& =6 n^{2}-10 n
\end{aligned}
$$

Now

$$
\operatorname{Spec}\left(K_{n-1, n-1}\right)=\left(\begin{array}{ccc}
n-1 & 0 & -(n-1) \\
1 & 2 n-4 & 1
\end{array}\right)
$$


and

$$
\operatorname{Spec}\left(K_{n}\right)=\left(\begin{array}{cc}
n-1 & -1 \\
1 & n-1
\end{array}\right) .
$$

By using (ii) of Lemma 5 and Theorem 1, $\operatorname{Spec}\left(\overline{K_{n-1, n-1} \otimes K_{n}}\right)=$

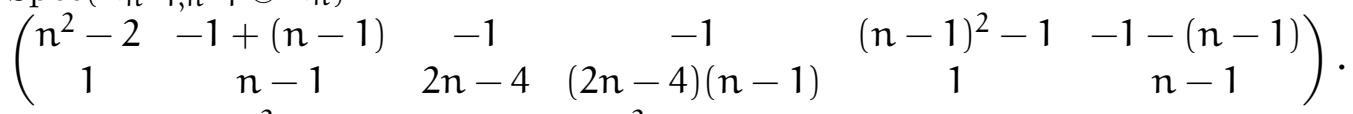

If $n \geq 3$ then $n^{2}-2, n-2$ and $(n-1)^{2}-1$ are only the positive $A$-eigenvalues of $\overline{K_{n-1, n-1} \otimes K_{n}}$. Therefore from definition of A-energy

$$
\begin{aligned}
E\left(\overline{K_{n-1, n-1} \otimes K_{n}}\right) & =2\left[n^{2}-2+(n-1)(n-2)+(n-1)^{2}-1\right] \\
& =6 n^{2}-10 n
\end{aligned}
$$

which completes the proof.

\subsection{Linear relations on energies of graphs}

Linear relations on energies of different graphs are not yet well studied in the study of graph energies except equienergetic graphs, that is $E\left(G_{1}\right)-E\left(G_{2}\right)=0$. In the following we present some linear relations on energies of different graphs of same order of the type $a E\left(G_{1}\right)+b E\left(G_{2}\right)=c$, where $a, b$ and $c$ are real numbers.

Theorem 17 If $\mathrm{n} \geq 3$ then $\mathrm{E}\left(\overline{\mathrm{L}\left(\mathrm{K}_{4}\right) \square \mathrm{K}_{\mathrm{n}}}\right)-\mathrm{E}\left(\mathrm{L}\left(\mathrm{K}_{4}\right) \square \mathrm{K}_{\mathrm{n}}\right)=2$.

Proof. We have

$$
\operatorname{Spec}\left(\mathrm{L}\left(\mathrm{K}_{4}\right)\right)=\left(\begin{array}{ccc}
4 & 0 & -2 \\
1 & 3 & 2
\end{array}\right)
$$

and

$$
\operatorname{Spec}\left(K_{n}\right)=\left(\begin{array}{cc}
n-1 & -1 \\
1 & n-1
\end{array}\right) .
$$

By using (i) of Lemma 5,

$$
\operatorname{Spec}\left(L\left(K_{4}\right) \square K_{n}\right)=\left(\begin{array}{cccccc}
n+3 & n-1 & n-3 & 3 & -1 & -3 \\
1 & 3 & 2 & n-1 & 3 n-3 & 2 n-2
\end{array}\right) .
$$

If $n \geq 3$ then -1 and -3 are only the negative $A$-eigenvalues of $L\left(K_{4}\right) \square K_{n}$. Therefore from definition of A-energy

$$
\begin{aligned}
E\left(L\left(K_{4}\right) \square K_{n}\right) & =2[(3 n-3)+3(2 n-2)] \\
& =18 n-18 .
\end{aligned}
$$


By using Theorem 1

$$
\operatorname{Spec}\left(\overline{\mathrm{L}\left(\mathrm{K}_{4}\right) \square \mathrm{K}_{n}}\right)=\left(\begin{array}{cccccc}
5 n-4 & -\mathrm{n} & 2-\mathrm{n} & -4 & 0 & 2 \\
1 & 3 & 2 & n-1 & 3 n-3 & 2 n-2
\end{array}\right) .
$$

If $n \geq 3$ then $-n, 2-n$ and -4 are only the negative $A$-eigenvalues of $\overline{\mathrm{L}\left(\mathrm{K}_{4}\right) \square \mathrm{K}_{\mathrm{n}}}$. Therefore from definition of A-energy

$$
\begin{aligned}
E\left(\overline{\mathrm{L}\left(\mathrm{K}_{4}\right) \square \mathrm{K}_{n}}\right) & =2[3 n+2 n-4+4 n-4] \\
& =18 n-16 .
\end{aligned}
$$

From (1) and (2) the result follows.

Theorem 18 If $n \geq 3$ then $\mathrm{E}\left(\mathrm{K}_{n-1, n-1} \otimes \mathrm{K}_{\mathrm{n}}\right)-\mathrm{E}\left(\mathrm{K}_{\mathrm{n}, \mathrm{n}} \otimes \mathrm{K}_{n-1}\right)=4$.

Proof. We have

$$
\operatorname{Spec}\left(K_{n, n}\right)=\left(\begin{array}{ccc}
n & 0 & -n \\
1 & 2 n-2 & 1
\end{array}\right)
$$

and

$$
\operatorname{Spec}\left(K_{n-1}\right)=\left(\begin{array}{cc}
n-2 & -1 \\
1 & n-2
\end{array}\right)
$$

By using (ii) of Lemma 5,

$\operatorname{Spec}\left(K_{n, n} \otimes K_{n-1}\right)=$

$$
\left(\begin{array}{cccccc}
n(n-2) & -n & 0 & 0 & -n(n-2) & n \\
1 & n-2 & 2 n-2 & (n-2)(2 n-2) & 1 & n-2
\end{array}\right) .
$$

If $n \geq 3$ then $n(n-2)$ and $n$ are only the positive $A$-eigenvalues of $K_{n, n} \otimes K_{n-1}$. Therefore from definition of A-energy

$$
\begin{aligned}
E\left(K_{n, n} \otimes K_{n-1}\right) & =2[n(n-2)+n(n-2)] \\
& =4 n^{2}-8 n .
\end{aligned}
$$

Now

$$
\operatorname{Spec}\left(K_{n-1, n-1}\right)=\left(\begin{array}{ccc}
n-1 & 0 & -(n-1) \\
1 & 2 n-4 & 1
\end{array}\right)
$$

and

$$
\operatorname{Spec}\left(K_{n}\right)=\left(\begin{array}{cc}
n-1 & -1 \\
1 & n-1
\end{array}\right) .
$$

By using (ii) of Lemma 5,

$\operatorname{Spec}\left(K_{n-1, n-1} \otimes K_{n}\right)=$ 
$\left(\begin{array}{cccccc}(n-1)^{2} & -(n-1) & 0 & 0 & -(n-1)^{2} & n-1 \\ 1 & n-1 & 2 n-4 & (n-2)(2 n-2) & 1 & n-1\end{array}\right)$.

If $n \geq 3$ then $(n-1)^{2}$ and $n-1$ are only the positive A-eigenvalues of $K_{n-1, n-1} \otimes K_{n}$. Therefore from definition of A-energy

$$
\begin{aligned}
E\left(K_{n-1, n-1} \otimes K_{n}\right) & =2\left[(n-1)^{2}+(n-1)^{2}\right] \\
& =4 n^{2}-8 n+4 .
\end{aligned}
$$

From (3) and (4) result follows.

Theorem 19 If $n \geq 3$ then

$$
E\left(K_{k \text { times }}^{n-1, n-1, \ldots, n-1} \otimes K_{n}\right)-E\left(K_{k \text { times }}^{K_{n, n, \ldots, n}} \nabla K_{n-1}\right)=2 .
$$

Proof. We have

$$
\operatorname{Spec}(\underbrace{K_{n, n, \ldots, n}}_{k \text { times }})=\left(\begin{array}{ccc}
n(k-1) & 0 & -n \\
1 & k(n-1) & k-1
\end{array}\right)
$$

and

$$
\operatorname{Spec}\left(K_{n-1}\right)=\left(\begin{array}{cc}
n-2 & -1 \\
1 & n-2
\end{array}\right)
$$

By using (iii) of Lemma 5 ,

$\operatorname{Spec}(\underbrace{n, n, \ldots, n}_{k \text { times }} \otimes K_{n-1})=$

$\left(\begin{array}{cccc}n^{2} k-n^{2}-n k+2 n-2 & n-2 & -1 & -n^{2}+2 n-2 \\ 1 & k(n-1) & k n(n-2) & k-1\end{array}\right)$.

If $n \geq 3$ then $n^{2} k-n^{2}-n k+2 n-2$ and $n-2$ are only the positive $A$-eigenvalues of $K_{k \text { times }}^{n, n, \ldots, n} \otimes K_{n-1}$. Therefore from definition of A-energy

$$
\begin{aligned}
E\left(K_{k \text { times }}^{n, n, \ldots, n} \otimes K_{n-1}\right) & =2\left[n^{2} k-n^{2}-n k+2 n-2+k(n-2)(n-1)\right] \\
& =4 n^{2} k-2 n^{2}-8 n k+4 n+4 k-4 .
\end{aligned}
$$

Now

$$
\operatorname{Spec}(\underbrace{K_{n-1, n-1, \ldots, n-1}}_{k \text { times }})=\left(\begin{array}{ccc}
(n-1)(k-1) & 0 & -(n-1) \\
1 & k(n-2) & k-1
\end{array}\right)
$$


and

$$
\operatorname{Spec}\left(K_{n}\right)=\left(\begin{array}{cc}
n-1 & -1 \\
1 & n-1
\end{array}\right)
$$

By using (iii) of Lemma 5 ,

$$
\begin{aligned}
& \operatorname{Spec}\left(K_{K_{\text {times }}^{n-1, n-1, \ldots, n-1}}^{n-K_{n}}\right)= \\
& \left(\begin{array}{cccc}
n^{2} k-n^{2}-n k+2 n-1 & n-1 & -1 & -n^{2}+2 n-1 \\
1 & k(n-2) & k(n-1)^{2} & k-1
\end{array}\right) .
\end{aligned}
$$

If $n \geq 3$ then $n^{2} k-n^{2}-n k+2 n-1$ and $n-1$ are only the positive $A$-eigenvalues

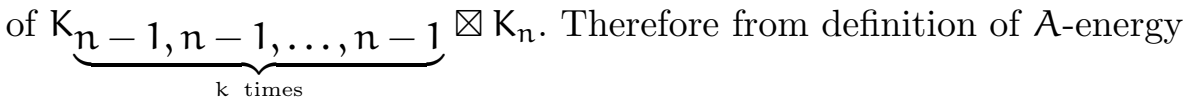

$$
\begin{aligned}
E(\underbrace{n-1, n-1, \ldots, n-1}_{k \text { times }} \otimes K_{n}) & =2\left[n^{2} k-n^{2}-n k+2 n-1+k(n-2)(n-1)\right] \\
& =4 n^{2} k-2 n^{2}-8 n k+4 n+4 k-2
\end{aligned}
$$

From (5) and (6) the result follows.

Theorem 20 If $\mathrm{n} \geq 3$ then $\mathrm{E}\left(\overline{\mathrm{K}_{n, n} \otimes \mathrm{K}_{n-1}}\right)-\mathrm{E}\left(\overline{\mathrm{K}_{n-1, n-1} \otimes \mathrm{K}_{n}}\right)=4$.

Proof. We have

$$
\operatorname{Spec}\left(K_{n, n}\right)=\left(\begin{array}{ccc}
n & 0 & -n \\
1 & 2 n-2 & 1
\end{array}\right)
$$

and

$$
\operatorname{Spec}\left(K_{n-1}\right)=\left(\begin{array}{cc}
n-2 & -1 \\
1 & n-2
\end{array}\right)
$$

By using (iii) of Lemma 5 and Theorem 1,

$$
\operatorname{Spec}\left(\overline{K_{n, n} \otimes K_{n-1}}\right)=\left(\begin{array}{ccc}
n^{2}-2 n+1 & 0 & -n+1 \\
2 & 2 n^{2}-2 n & 2 n-2
\end{array}\right) .
$$

If $n \geq 3$ then $n^{2}-2 n+1$ is only the positive $A$-eigenvalues of $\overline{K_{n, n} \otimes K_{n-1}}$. Therefore from definition of A-energy

$$
\begin{aligned}
E\left(\overline{K_{n, n} \otimes K_{n-1}}\right) & =2\left[2 n^{2}-4 n+2\right] \\
& =4 n^{2}-8 n+4 .
\end{aligned}
$$

Now

$$
\operatorname{Spec}\left(K_{n-1, n-1}\right)=\left(\begin{array}{ccc}
n-1 & 0 & -(n-1) \\
1 & 2 n-4 & 1
\end{array}\right)
$$


and

$$
\operatorname{Spec}\left(K_{n}\right)=\left(\begin{array}{cc}
n-1 & -1 \\
1 & n-1
\end{array}\right)
$$

By using (iii) of Lemma 5 and Theorem 1,

$$
\operatorname{Spec}\left(\overline{K_{n-1, n-1} \otimes K_{n}}\right)=\left(\begin{array}{ccc}
n^{2}-2 n & 0 & -n \\
2 & 2 n^{2}-4 n+2 & 2 n-4
\end{array}\right) .
$$

If $n \geq 3$ then $n^{2}-2 n$ is only the positive $A$-eigenvalues of $\overline{K_{n-1, n-1} \otimes K_{n}}$. Therefore from definition of A-energy

$$
\begin{aligned}
E\left(\overline{K_{n-1, n-1} \otimes K_{n}}\right) & =2\left[2 n^{2}-4 n\right] \\
& =4 n^{2}-8 n .
\end{aligned}
$$

From (7) and (8) the result follows.

\section{S-equienergetic graphs and linear relations on S- energies of certain class of graphs}

\subsection{S-equienergetic graphs}

In [16] Ramane et al. studied S-energy of $\mathrm{L}^{2}(\mathrm{G})$ for an $r$-regular graph $\mathrm{G}, \mathrm{r} \geq 3$ and constructed a large class of $S$-equienergetic graphs. The following result provides $S$-equienergetic graphs with the help of iterated line graphs $L^{k}(G)$ even for $k \geq 1$, where $L^{0}(G)=G$.

Theorem 21 If $\mathrm{n} \geq 5, \mathrm{k} \geq 0$ then $\mathrm{E}_{\mathrm{S}}\left(\mathrm{L}^{\mathrm{k}}\left(\mathrm{K}_{\mathrm{n}, \mathrm{n}} \square \mathrm{K}_{\mathrm{n}-1}\right)\right)=\mathrm{E}_{S}\left(\mathrm{~L}^{\mathrm{k}}\left(\mathrm{K}_{\mathrm{n}-1, \mathrm{n}-1} \square \mathrm{K}_{\mathrm{n}}\right)\right)$.

Proof. As $K_{n, n} \square K_{n-1}$ and $K_{n-1, n-1} \square K_{n}$ are both regular graphs of same order and of same degree, by Theorems 3 and 4 , the result is true for $k \geq 2$. Now, it is enough to prove for $k=0,1$.

When $k=0$.

$$
\operatorname{Spec}\left(K_{n, n}\right)=\left(\begin{array}{ccc}
n & 0 & -n \\
1 & 2 n-2 & 1
\end{array}\right)
$$

and

$$
\operatorname{Spec}\left(K_{n-1}\right)=\left(\begin{array}{cc}
n-2 & -1 \\
1 & n-2
\end{array}\right)
$$


Therefore by (i) of Lemma 5 ,

$\operatorname{Spec}\left(K_{n, n} \square K_{n-1}\right)=\left(\begin{array}{cccccc}2 n-2 & n-1 & n-2 & -1 & -2 & -n-1 \\ 1 & n-2 & 2 n-2 & (2 n-2)(n-2) & 1 & n-2\end{array}\right)$

and

$\operatorname{Spec}\left(K_{n-1, n-1} \square K_{n}\right)=\left(\begin{array}{cccccc}2 n-2 & n-2 & n-1 & -1 & 0 & -n \\ 1 & n-1 & 2 n-4 & (2 n-4)(n-1) & 1 & n-1\end{array}\right)$.

Therefore by Theorem 3,

$\operatorname{Spec}_{S}\left(K_{n, n} \square K_{n-1}\right)=$

$$
\left(\begin{array}{cccccc}
2 n^{2}-6 n+3 & 1-2 n & 3-2 n & 1 & 3 & 2 n+1 \\
1 & n-2 & 2 n-2 & (2 n-2)(n-2) & 1 & n-2
\end{array}\right)
$$

and $\operatorname{Specs}_{S}\left(K_{n-1, n-1} \square K_{n}\right)=$

$$
\left(\begin{array}{cccccc}
2 n^{2}-6 n+3 & 3-2 n & 1-2 n & 1 & -1 & 2 n-1 \\
1 & n-1 & 2 n-4 & (2 n-4)(n-1) & 1 & n-1
\end{array}\right)
$$

If $n \geq 5$ then $2 n^{2}-6 n+3,1,3$ and $2 n+1$ are only the positive $S$-eigenvalues of $K_{n, n} \square K_{n-1}$. Therefore from definition of S-energy

$$
\begin{aligned}
E_{S}\left(K_{n, n} \square K_{n-1}\right) & =2\left[2 n^{2}-6 n+3+(2 n-2)(n-2)+3+(n-2)(2 n+1)\right] \\
& =12 n^{2}-26 n+16 .
\end{aligned}
$$

If $n \geq 5$ then $2 n^{2}-6 n+3,1$ and $2 n-1$ are only the positive $S$-eigenvalues of $K_{n-1, n-1} \square K_{n}$. Therefore from definition of S-energy

$$
\begin{aligned}
E_{S}\left(K_{n-1, n-1} \square K_{n}\right) & =2\left[2 n^{2}-6 n+3+(2 n-4)(n-1)+(n-1)(2 n-1)\right] \\
& =12 n^{2}-26 n+16 .
\end{aligned}
$$

Hence $E_{S}\left(K_{n, n} \square K_{n-1}\right)=E_{S}\left(K_{n-1, n-1} \square K_{n}\right)$.

When $k=1$ both $K_{n, n} \square K_{n-1}$ and $K_{n-1, n-1} \square K_{n}$ are regular graphs of same order $2 n(n-1)$ and of same degree $2 n-2$. Hence by (i) of Lemma 5 , $\operatorname{Spec}\left(\mathrm{L}\left(\mathrm{K}_{\mathrm{n}, \mathrm{n}} \square \mathrm{K}_{\mathrm{n}-1}\right)\right)=$

$\left(\begin{array}{ccccccc}4 n-6 & 3 n-5 & 3 n-6 & 2 n-5 & 2 n-6 & n-5 & -2 \\ 1 & n-2 & 2 n-2 & (2 n-2)(n-2) & 1 & n-2 & 2 n(n-1)(n-2)\end{array}\right)$ 
and $\operatorname{Spec}\left(\mathrm{L}\left(\mathrm{K}_{\mathrm{n}-1, \mathrm{n}-1} \square \mathrm{K}_{\mathrm{n}}\right)\right)=$

$\left(\begin{array}{ccccccc}4 n-6 & 3 n-6 & 3 n-5 & 2 n-5 & 2 n-4 & n-4 & -2 \\ 1 & n-1 & 2 n-4 & (2 n-4)(n-1) & 1 & n-1 & 2 n(n-1)(n-2)\end{array}\right)$.

Therefore from Theorem 3,

$\operatorname{Spec}_{S}\left(L\left(K_{n, n} \square K_{n-1}\right)\right)=$

$$
\begin{aligned}
& \left(\begin{array}{cccc}
2 n(n-1)^{2}-8 n+11 & 9-6 n & 11-6 n & 9-4 n \\
1 & n-2 & 2 n-2 & (2 n-2)(n-2)
\end{array}\right. \\
& \left.\begin{array}{ccc}
11-4 n & 9-2 n & 3 \\
1 & n-2 & 2 n(n-1)(n-2)
\end{array}\right)
\end{aligned}
$$

and $\operatorname{Spec}_{S}\left(L\left(K_{n-1, n-1} \square K_{n}\right)\right)=$

$$
\begin{aligned}
& \left(\begin{array}{cccc}
2 n(n-1)^{2}-8 n+11 & 11-6 n & 9-6 n & 9-4 n \\
1 & n-1 & 2 n-4 & (2 n-4)(n-1)
\end{array}\right. \\
& \left.\begin{array}{ccc}
7-4 n & 7-2 n & 3 \\
1 & n-1 & 2 n(n-1)(n-2)
\end{array}\right)
\end{aligned}
$$

If $n \geq 5$ then $2 n(n-1)^{2}-8 n+11$ and 3 are only the positive $S$-eigenvalues of $L\left(K_{n, n} \square K_{n-1}\right)$. Therefore from definition of S-energy

$$
\begin{aligned}
\mathrm{E}_{S}\left(\mathrm{~L}\left(\mathrm{~K}_{\mathrm{n}, \mathrm{n}} \square \mathrm{K}_{n-1}\right)\right) & =2\left[2 \mathrm{n}(\mathrm{n}-1)^{2}-8 \mathrm{n}+11+6 \mathrm{n}\left(\mathrm{n}^{2}-3 \mathrm{n}+2\right)\right] \\
& =16 \mathrm{n}^{3}-44 n^{2}+18 \mathrm{n}+22 .
\end{aligned}
$$

If $n \geq 5$ then $2 n(n-1)^{2}-8 n+11$ and 3 are only the positive $S$-eigenvalues of $L\left(K_{n, n} \square K_{n-1}\right)$. Therefore from definition of S-energy

$$
\begin{aligned}
E_{S}\left(L\left(K_{n, n} \square K_{n-1}\right)\right) & =2\left[2 n(n-1)^{2}-8 n+11+6 n\left(n^{2}-3 n+2\right)\right] \\
& =16 n^{3}-44 n^{2}+18 n+22 .
\end{aligned}
$$

Hence $\mathrm{E}_{\mathrm{S}}\left(\mathrm{L}\left(\mathrm{K}_{\mathrm{n}, \mathrm{n}} \square \mathrm{K}_{\mathrm{n}-1}\right)\right)=\mathrm{E}_{\mathrm{S}}\left(\mathrm{L}\left(\mathrm{K}_{\mathrm{n}-1, \mathrm{n}-1} \square \mathrm{K}_{\mathrm{n}}\right)\right)$.

Theorem 22 If $n \geq 3$ then $\mathrm{E}_{S}\left(\mathrm{~K}_{\mathrm{n}, \mathrm{n}} \otimes \mathrm{K}_{\mathrm{n}-1}\right)=\mathrm{E}_{S}\left(\mathrm{~K}_{\mathrm{n}-1, \mathrm{n}-1} \otimes \mathrm{K}_{\mathrm{n}}\right)$.

Proof. We have

$$
\operatorname{Spec}\left(K_{n, n}\right)=\left(\begin{array}{ccc}
n & 0 & -n \\
1 & 2 n-2 & 1
\end{array}\right)
$$


and

$$
\operatorname{Spec}\left(K_{n-1}\right)=\left(\begin{array}{cc}
n-2 & -1 \\
1 & n-2
\end{array}\right)
$$

By using (iii) of Lemma 5 and Theorem 3,

$$
\operatorname{Spec}_{S}\left(K_{n, n} \otimes K_{n-1}\right)=\left(\begin{array}{cccc}
-2 n+3 & 1 & 2 n^{2}-4 n+3 & -2 n+3 \\
1 & 2 n^{2}-2 n & 1 & 2 n-2
\end{array}\right) .
$$

If $n \geq 3$ then $2 n^{2}-4 n+3$ and 1 are only the positive S-eigenvalues of $K_{n, n} \otimes K_{n-1}$. Therefore from definition of S-energy

$$
\begin{aligned}
E_{S}\left(K_{n, n} \otimes K_{n-1}\right) & =2\left[2 n^{2}-2 n+2 n^{2}-4 n+3\right] \\
& =8 n^{2}-16 n+6 .
\end{aligned}
$$

Now

$$
\operatorname{Spec}\left(K_{n-1, n-1}\right)=\left(\begin{array}{ccc}
n-1 & 0 & -(n-1) \\
1 & 2 n-4 & 1
\end{array}\right)
$$

and

$$
\operatorname{Spec}\left(K_{n}\right)=\left(\begin{array}{cc}
n-1 & -1 \\
1 & n-1
\end{array}\right)
$$

By using (iii) of Lemma 5 and Theorem 3,

$\operatorname{Spec}_{S}\left(K_{n-1, n-1} \otimes K_{n}\right)=\left(\begin{array}{cccc}-2 n+1 & 1 & 2 n^{2}-4 n+1 & -2 n+1 \\ 1 & (n-1)(2 n-2) & 1 & 2 n-4\end{array}\right)$.

If $n \geq 5$ then $2 n^{2}-4 n+1$ and 1 are only the positive S-eigenvalues of $K_{n-1, n-1} \otimes K_{n}$. Therefore from definition of S-energy

$$
\begin{aligned}
E_{S}\left(K_{n-1, n-1} \otimes K_{n}\right) & =2\left[(n-1)(2 n-2)+2 n^{2}-4 n+1\right] \\
& =8 n^{2}-16 n+6
\end{aligned}
$$

which completes the proof.

\subsection{Linear relations on S-energies of graphs}

Linear relations on S-energies of different graphs are not yet well studied in the study of $S$-energies. In the following we present some linear relations on S-energies of different graphs of same order of the type $a E_{S}\left(G_{1}\right)+b E_{S}\left(G_{2}\right)=c$, where $a, b$ and $c$ are real numbers. 
Theorem 23 If $n \geq 3$ then $\mathrm{E}_{S}\left(\mathrm{~K}_{n-1, n-1} \otimes \mathrm{K}_{n}\right)-\mathrm{E}_{S}\left(\mathrm{~K}_{\mathrm{n}, \mathrm{n}} \otimes \mathrm{K}_{\mathrm{n}-1}\right)=2$.

Proof. We have

$$
\operatorname{Spec}\left(K_{n, n}\right)=\left(\begin{array}{ccc}
n & 0 & -n \\
1 & 2 n-2 & 1
\end{array}\right)
$$

and

$$
\operatorname{Spec}\left(K_{n-1}\right)=\left(\begin{array}{cc}
n-2 & -1 \\
1 & n-2
\end{array}\right)
$$

By using (ii) of Lemma 5 and Theorem 3,

$\operatorname{Spec}_{S}\left(K_{n, n} \otimes K_{n-1}\right)=\left(\begin{array}{cccc}2 n-1 & -1-2 n & -1 & 2 n(n-2)-1 \\ n-1 & n-2 & (n-1)(2 n-2) & 1\end{array}\right)$.

If $n \geq 5$ then $2 n-1$ and $2 n(n-2)-1$ are only the positive $S$-eigenvalues of $K_{n, n} \otimes K_{n-1}$. Therefore from definition of S-energy

$$
\begin{aligned}
E_{S}\left(K_{n, n} \otimes K_{n-1}\right) & =2[(2 n-1)(n-1)+2 n(n-2)-1] \\
& =8 n^{2}-14 n .
\end{aligned}
$$

Now

$$
\operatorname{Spec}\left(K_{n-1, n-1}\right)=\left(\begin{array}{ccc}
n-1 & 0 & -(n-1) \\
1 & 2 n-4 & 1
\end{array}\right)
$$

and

$$
\operatorname{Spec}\left(K_{n}\right)=\left(\begin{array}{cc}
n-1 & -1 \\
1 & n-1
\end{array}\right)
$$

By using (ii) of Lemma 5 and Theorem 3,

$$
\operatorname{Specs}_{S}\left(K_{n-1, n-1} \otimes K_{n}\right)=\left(\begin{array}{cccc}
2 n-3 & 1-2 n & -1 & 2 n^{2}-4 n+1 \\
n & n-1 & n(2 n-4) & 1
\end{array}\right) .
$$

If $n \geq 5$ then $2 n-3$ and $2 n^{2}-4 n+1$ are only the positive $S$-eigenvalues of $K_{n-1, n-1} \otimes K_{n}$. Therefore from definition of S-energy

$$
\begin{aligned}
E_{S}\left(K_{n-1, n-1} \otimes K_{n}\right) & =2\left[n(2 n-3)+2 n^{2}-4 n+1\right] \\
& =8 n^{2}-14 n+2 .
\end{aligned}
$$

From (9) and (10) the result follows. 


\section{Conclusion}

In this article we have obtained several classes of A-equienergetic and Sequienergetic graphs by using Cartesian product, tensor product and strong product. The results can be further extended to the other class of graphs. Also some linear relations between $A$-energies and S-energies of graphs has been established which shows a possible new direction in the study of relation between energies of different graphs of same order.

\section{Acknowledgements}

The authors would like to thank the anonymous referees for their helpful suggestions.

\section{References}

[1] C. Adiga, B. R. Rakshith, On spectra of variants of the corona of two graphs and some new equienergetic graphs, Discuss. Math. Graph Theory 36 (2016) $127-140 . \Rightarrow 197$

[2] R. Balakrishnan, The energy of a graph, Linear Algebra Appl. 387 (2004) 287295. $\Rightarrow 197$

[3] A. S. Bonifácio, C. T. M. Vinagre, N. M. M. de Abreu, Constructing pairs of equienergetic and non-cospectral graphs, Appl. Math. Lett. 21 (2008) 338-341. $\Rightarrow 197$

[4] V. Brankov, D. Stevanović, I. Gutman, Equienergetic chemical trees, J. Serb. Chem. Soc. 69 (2004) 549-553. $\Rightarrow 197$

[5] A. E. Brouwer, W. H. Haemers, Spectra of Graphs, Springer, Berlin, $2012 . \Rightarrow$ 198

[6] D. Cvetković, P. Rowlinson, S. Simić, An Introduction to the Theory of Graph Spectra, Cambridge University Press, Cambridge, 2010. $\Rightarrow 197,198$

[7] H. A. Ganie, S. Pirzada, A. Iványi, Energy, Laplacian energy of double graphs and new families of equienergetic graphs, Acta Univ. Sapientiae, Informatica. 6 (2014) 89-116. $\Rightarrow 197$

[8] I. Gutman, The energy of a graph, Ber. Math. Stat. Sekt. Forschungszentrum Graz. 103 (1978) 1-22. $\Rightarrow 197$

[9] W. H. Haemers, Seidel switching and graph energy, MATCH Commun. Math. Comput. Chem. 68, 3 (2012) 653-659. $\Rightarrow 197$

[10] F. Harary, Graph Theory, Narosa Publishing House, New Delhi, 1998. $\Rightarrow 196$

[11] G. Indulal, A.Vijayakumar, On a pair of equienergetic graphs, MATCH Commun. Math. Comput. Chem. 55, 1 (2006) 83-90. $\Rightarrow 197$ 
[12] J. Liu, B. Liu, Note on a pair of equienergetic graphs, MATCH Commun. Math. Comput. Chem. 59, 2 (2008) 275-278. $\Rightarrow 197$

[13] O. Miljković, B. Furtula, S. Radenković, I. Gutman, Equienergetic and almost equienergetic trees, MATCH Commun. Math. Comput. Chem. 61, 2 (2009) 451461. $\Rightarrow 197$

[14] H. S. Ramane, I. Gutman, H. B. Walikar, S. B. Halkarni, Equienergetic complement graphs, Kragujevac J. Sci. 27 (2005) 67-74. $\Rightarrow 197$

[15] H. S. Ramane, M. M. Gundloor, S. M. Hosamani, Seidel equienergetic graphs, Bull. Math. Sci. Appl. 16 (2016) 62-69. $\Rightarrow 197$

[16] H. S. Ramane, I. Gutman, M. M. Gundloor, Seidel energy of iterated line graphs of regular graphs, Kragujevac J. Math. 39, 1 (2015) 7-12. $\Rightarrow 198,213$

[17] H. S. Ramane, B. Parvathalu, D. D. Patil, K. Ashoka, Graphs equienergetic with their complements, MATCH Commun. Math. Comput. Chem. 82, 2 (2019) 471-480. $\Rightarrow 197,198,203$

[18] H. S. Ramane, K. Ashoka, D. Patil, B. Parvathalu, I. Gutman, On complementary equienergetic strongly regular graphs, Discrete Math. Lett. 4 (2020) 50-55. $\Rightarrow 197$

[19] H. S. Ramane, D. Patil, K. Ashoka, B. Parvathalu, Equienergetic graphs using Cartesian product and generalized composition, Sarajevo J. Math. 17, 30 No.1 (2021) $7-21 . \Rightarrow 200$

[20] H. S. Ramane, H. B. Walikar, Construction of equienergetic graphs, MATCH Commun. Math. Comput. Chem. 57, 1 (2007) 203-210. $\Rightarrow 197$

[21] H. S. Ramane, H. B. Walikar, S. B. Rao, B. D. Acharya, P. R. Hampiholi, S. R. Jog, I. Gutman, Spectra and energies of iterated line graphs of regular graphs, Appl. Math. Lett. 18 (2005) 679-682. $\Rightarrow 197$

[22] I. Stanković, M. Milošević, D. Stevanović, Small and not so small equienergetic graphs, MATCH Commun. Math. Comput. Chem. 61, 2 (2009) 443-450. $\Rightarrow 197$

[23] D. Stevanović, Energy and NEPS of graphs, Linear Multilinear Algebra 53 (2005) $67-74 . \Rightarrow 197$

[24] S. K. Vaidya, K. M. Popat, Some new results on Seidel equienergetic graphs, Kyungpook Math. J. 59, 2 (2019) 335-340. $\Rightarrow 197$

Received: June 2. 2021 • Revised: August 18, 2021 